Cahiers d'études africaines

169-170|2003

Enseignements

\title{
Retour vers les dugsi, écoles coraniques en Somalie
}

Mohamed Mohamed-Abdi

\section{OpenEdition}

Journals

Édition électronique

URL : http://journals.openedition.org/etudesafricaines/204

DOI : 10.4000/etudesafricaines.204

ISSN : 1777-5353

Éditeur

Éditions de l'EHESS

Édition imprimée

Date de publication : 1 janvier 2003

Pagination : 351-369

ISBN : 978-2-7132-1809-5

ISSN : 0008-0055

Référence électronique

Mohamed Mohamed-Abdi, "Retour vers les dugsi, écoles coraniques en Somalie », Cahiers d'études africaines [En ligne], 169-170 | 2003, mis en ligne le 21 décembre 2006, consulté le 19 avril 2019. URL http://journals.openedition.org/etudesafricaines/204; DOI : 10.4000/etudesafricaines.204 


\section{Mohamed Mohamed-Abdi}

\section{Retour vers les dugsi, écoles coraniques en Somalie}

La République de Somalie n'a plus aujourd'hui de réalité politique. Il n'y a plus dans ce pays ni gouvernement ni administration depuis l'éclatement de la guerre civile et donc aucune politique scolaire. Avec l'aide humanitaire internationale, quelques écoles, parrainées par l'Unesco, l'Unicef et des ONG ont vu le jour, en particulier dans les camps de réfugiés. Mais, sur la majeure partie du territoire, aucune infrastructure officielle n'assure l'éducation des enfants et des adolescents.

Des initiatives privées sans soutien extérieur permettent toutefois aux enfants d'accéder à un minimum de connaissances. Certaines, laïques, ont été mises en place par des organisations somaliennes militant pour la paix. D'autres, religieuses, constituent à l'échelle nationale une résurgence des écoles coraniques traditionnelles. Mais tandis que les premières ont pour objectif l'apprentissage d'un savoir minimal en langue somali (lecture, écriture, règles de la langue et calcul), les secondes limitent leur enseignement à l'apprentissage du Coran et à l'écriture de quelques versets.

La société civile prend donc en charge une partie de l'éducation de la jeunesse et, réinvestie de sa fonction éducative, perpétue les pratiques de transmission du savoir. On peut voir là une volonté de ne pas interrompre la relation entre les générations contenue dans l'enseignement, mais il y a sans doute aussi impossibilité de combler le vide induit par la guerre civile et le « choc des cultures ».

Dans l'hypothèse de la restauration d'un État de droit en Somalie et du retour au pays d'éléments de la diaspora, on peut d'ores et déjà craindre un nouveau choc des cultures et choc des savoirs entre les jeunes adultes éduqués au sein de la société civile avec peu de moyens et ceux qui auront pu suivre une scolarité complète à l'étranger (principalement dans les pays occidentaux).

Précisons qu'il n'existe quasiment aucune donnée statistique concernant les écoles coraniques en territoire somali. Les seules à ma disposition ont été recueillies dans les publications de Hamdi Al-Sayid Salim (1965) et du 
Haut comité national pour la campagne de renforcement de la langue arabe ${ }^{1}$ (1986) et encore sont-elles partielles. De même, les données sur l'enseignement public ou privé, pendant la période coloniale puis postcoloniale, sont peu nombreuses et bien souvent contradictoires, et elles restent lacunaires.

\section{Écoles coraniques traditionnelles}

Du fait de leur proximité, les côtes de la péninsule arabe et celles de la péninsule somali ont entretenu dès l'Antiquité des relations étroites. L'islam a donc été introduit très tôt dans cette région de l'Afrique et s'y est diffusé progressivement. C'est aujourd'hui la religion de tous les Somali².

\section{L'école au quotidien}

L'enseignement coranique est un des piliers de l'islam. Dispensé dans les écoles coraniques traditionnelles (dugsi ou malcaamad) aux seuls garçons, il est limité à l'apprentissage par cœur du Coran (lecture, écriture), sans l'appui d'aucun livre. Le maître lui-même n'en utilise pas dans sa classe. L'apprentissage est organisé en deux cycles correspondant à la division du Coran en deux parties (awal et akhir), chacune partagée en plusieurs juz (jus en somali). Chaque jus se compose de deux ahzâb (chapitres). La progression normale d'apprentissage est dite hor-u-jeed. Si un enfant, capable de lire et d'écrire le Coran sous la dictée, ne peut le réciter par cœur dans son ensemble, il recommence alors un apprentissage accéléré, appelé naqtiin.

L'École coranique est une école de proximité ; c'est semble-t-il une constante dans l'ensemble des pays musulmans (Meunier 1995 : 620 ; Tourneux \& Iyébi-Mandjek 1994 : 88). Dans les villes, elle peut se tenir dans une cour ou un jardin privé, ou encore, à la croisée de plusieurs chemins. C'est un cercle bordé de claies tressées, situé sous un grand arbre, ou recouvert partiellement de nattes de paille. Il n'y a aucun meuble sur lequel s'asseoir ou écrire, et seul le maître peut bénéficier d'une natte (darin) ou d'un tabouret (gambar) disposé dans un lieu légèrement surélevé. Il n'y a pas non plus de tableau. Les cours débutent à l'aube, après la prière du matin (salaat as-subhi) et finissent après la prière du soir (makhrib). La journée est coupée par deux pauses (vers huit heures et vers midi) d'environ une heure pendant lesquelles les enfants rentrent chez eux prendre un repas ou

1. Le nom arabe de ce comité est : Lujna al wataniya al uliya li hamlat taqwiyati al luqa al arabiya. Dans la suite du texte, nous le citerons en abrégé : Lujna.

2. Les Somali (appartenant à l'aire culturelle somali, c'est-à-dire République de Somalie, province du Nord-Est au Kenya, province de l'Ogaden en Éthiopie et moitié méridionale du territoire djiboutien) sont à $99 \%$ musulmans sunnites, de rite shafi'ite. 
une collation, la seconde pause étant suivie d'une sieste obligatoire (d'environ une heure) dans l'enclos. Les cours reprennent ensuite jusqu'à la prière makhrib. Après celle-ci, les enfants récitent une bénédiction (shaafiin) et rentrent chez eux. Les mercredis et jeudis après-midi, ils ramassent ensemble du bois mort (xaabo) pour les flambées du matin et les veillées. Le seul jour férié de la semaine est le vendredi.

Le matériel de l'élève (arday) est rudimentaire : une planche de bois, rectangulaire avec une poignée triangulaire à chaque extrémité, taillée dans du bois de dharkayn ou de xoday, sert de tablette (loox). Elle est polie avec des galets $($ sulub) puis frottée avec des herbes afin de l'imperméabiliser et d'éviter que l'encre ne pénètre le bois. L'encre (inqaas) est obtenue en mélangeant dans un petit récipient à fond rugueux de l'eau, de la myrrhe (malmal) en poudre et du charbon de bois (dhuxul) effrité sur le fond (ou à défaut, du lait et du charbon). Lorsque le mélange atteint la consistance voulue, il est versé dans un encrier (tawaad). Une baguette dure, taillée en biseau dans une branche de duur, sert de stylet (qalin).

L'École coranique est payante. L'inscription (fadhiisin), faite par le père, est réglée par le don d'une chèvre, mais en ville, c'est une somme d'argent qui est versée. L'apprentissage s'étale sur une période de deux à six ans, voire neuf ans, selon les capacités intellectuelles de l'enfant et les moyens économiques des parents. Un enfant peut être admis à l'École coranique dès l'âge de quatre ans, mais le véritable apprentissage débute vers l'âge de six-sept ans. Lorsque l'enfant achève l'awal, son père paie le maître avec une grosse tête de bétail (dromadaire ou vache, ou l'équivalent en petit bétail ou en argent): il commence alors le second cycle (akhir). À la fin de ce second cycle, son père donne alors une autre tête de bétail au maître. Lewis (1961 : 215) indique des prix beaucoup plus élevés : un agneau pour chacune des trente jus apprise et un dromadaire en fin d'apprentissage. En ville, le paiement peut être hebdomadaire : à jour fixe (le mercredi le plus souvent), le père verse une somme symbolique au macallin et le salaire convenu en fin de mois. L'enfant qui a appris par cœur l'ensemble du Coran est dit macallin (maître); il est gardien du Coran (xaafid, forme somalisée de hafiz).

\section{Statuts du maître et des élèves}

Certains maîtres (macallin), sédentaires, exercent leur métier dans une mosquée ou à proximité de la tombe d'un saint musulman dont ils ont la charge. D'autres, itinérants, voyagent en compagnie de leurs novices, exécutant les cérémonies à la demande de leurs hôtes. Un certain nombre, enfin, suivent leurs élèves avec leurs familles, étant membres du même groupe social. L'effectif des classes est très variable : d'une dizaine d'élèves dans les campements itinérants jusqu'à quatre-vingts, de niveaux différents, dans les villages fixes et les villes. Le maître reçoit de la communauté et des parents 
une considération qui croît avec l'âge, le nombre d'écoles qu'il a fondées et les générations d'enfants qu'il a éduquées. Il est autorisé à punir les enfants, parfois avec sévérité mais sans dépasser certaines limites. Chaque enfant participe au ramassage hebdomadaire des verges souples avec lesquelles le maître peut le châtier. L'élève brillant est félicité, tant par ses camarades que par le maître et son entourage. Les parents n'hésitent d'ailleurs pas à organiser une petite fête, dite bunayn (frire le café), avec repas amélioré et félicitations de la famille proche, lorsque l'enfant a achevé l'apprentissage d'une jus. La fin d'un cycle complet est célébrée en famille, avec les voisins et les camarades de classe. Quand les parents ont pris la décision de placer un enfant à l'École coranique, il est dispensé d'une partie des travaux quotidiens (garde des troupeaux de petit bétail, ramassage du bois pour la maison, etc.).

\section{Techniques d'apprentissage}

L'apprentissage commence avec la lecture et l'écriture de l'alphabet arabe : le maître copie sur la tablette les lettres et les mots que le débutant doit apprendre à son rythme. Au fur et à mesure qu'il copie les lettres, il les prononce plusieurs fois pour que l'enfant enregistre leur prononciation et associe un symbole au son. L'alphabet est enseigné à l'aide d'une méthode mnémotechnique en langue somali (higgaad) transmise au XII ${ }^{\mathrm{e}}$ siècle par le cheikh somali Sheekh Yuusuf al Kawneyn, dit aussi Aw Barkhadle, et adaptée de la mnémotechnie arabe. Cette méthode se compose de plusieurs étapes. Pour que l'enfant s'habitue aux sons de la langue arabe, il doit apprendre à nommer les vingt-huit lettres de l'alphabet et à les reconnaître. Puis il les reprend en mémorisant la façon de les calligraphier - c'est le alif wax ma leh: alif wax ma leh (alif n'a rien), ba hoos ku hal leh (ba un point dessous), ta kor ku laba leh (ta deux points au-dessus), etc. (Mohamed-Abdi 1990: 508). Ensuite vient l'étape du alif la kor dhabey (alif un trait en haut): écriture et prononciation des syllabes courtes où interviennent les signes diacritiques fatha (a), kasra (i) et dhumma (u). Puis intervient l'étape du alif dheer pour les syllabes longues qui font appel au diacritique sukun (fermeture). En dernier, on apprend la calligraphie alif laba koor dhaban des accentuations toniques (doublement des signes diacritiques). Chaque leçon est assimilée en combinant écriture et déclamation : en même temps que l'enfant écrit une lettre ou une syllabe, il chante la façon de l'écrire. La mélopée monocorde est rythmée. Les débutants doivent aussi réécrire de mémoire les mots et les sons appris (feegaar). Des dictées de sons, de mots et de combinaisons de syllabes sont faites régulièrement pour permettre au maître de vérifier que l'enfant maîtrise l'écriture : c'est le $a b$ jed. C'est seulement à partir de ce moment que l'élève commence l'apprentissage du Coran lui-même. Comme les élèves coraniques de l'Afrique occidentale (Meunier 1997 : 119-122), il débute avec la formule 
Bismillahi... que l'on récite avant chaque sourate et au début de chaque prière. Le maître énonce chaque lettre de la formule, en précisant son écriture. L'élève apprend ensuite la Fatiha (Prologue, la $1^{\text {re }}$ sourate), clé de toutes les prières, puis l'An-nas (Les Hommes, la 114 ${ }^{\mathrm{e}}$ sourate), et ensuite en remontant le Coran, c'est-à-dire en apprenant les sourates dans l'ordre décroissant de leur numérotation, jusqu'à la deuxième (Al-baqara: la Génisse). Lorsqu'il connaît par cœur les sourates 1 et 105 (El-Fil : l'Éléphant) à 114 , il peut participer aux prières communes ou prier seul.

\section{Organisation d'une journée à l'école coranique}

Les enfants arrivent à l'école à l'aube (oog) et allument un feu qui sert tant à l'éclairage qu'à les réchauffer. Pendant que certains élèves apprennent ce qui leur a été dicté précédemment, d'autres récitent leur leçon à tour de rôle. Lorsqu'elle est sue, ils lavent leur tablette pour une nouvelle leçon. Une séance de dictée (yeerin) se déroule ainsi : les enfants sont placés en cercle autour du maître, un premier enfant énonce le dernier verset qu'il a appris et le maître lui dicte la suite ; pendant qu'il écrit, un autre élève, apprenant un autre verset, indique au maître où il s'est arrêté dans sa copie, et ainsi de suite pour l'ensemble des élèves. Le maître dicte ainsi quasi simultanément une vingtaine de versets tirés de sourates différentes. La dictée d'un élève s'achève lorsque sa tablette est remplie de lignes d'écriture. Le maître vérifie alors ce qui a été écrit (sixid), efface les erreurs avec le stylet trempé dans l'encre et l'enfant essuie l'encre mouillée avec son doigt puis le frotte sur sa joue, car «les paroles sacrées ne doivent pas être jetées n'importe où » et le maître corrige sa «copie». Il ajoute au besoin les intonations (signes diacritiques). L'enfant s'écarte des autres pour apprendre par cœur le texte qu'il ne comprend pas. Lorsqu'il pense le connaître, il se présente devant le maître avec sa tablette : il s'assied, tient devant lui la tablette tournée vers le maître et récite ce qui y est écrit ( $k a$ bixid). S'il se trompe, il est puni et renvoyé dans un coin pour continuer à apprendre. Dans le cas contraire, le maître l'envoie laver sa tablette et lui dicte une nouvelle leçon. Les enfants des familles aisées ont plusieurs tablettes, utilisées à tour de rôle. Chacune d'elles est couverte d'une leçon, à divers stades d'apprentissage ; la plus ancienne est effacée lorsque son contenu est su, afin d'en recopier une nouvelle. L'élève le plus avancé et le plus sage est désigné par le maître comme kabiir (assistant) : il fait réciter les plus jeunes et les aide dans l'apprentissage de la lecture et de l'écriture, il surveille la classe en l'absence du maître et participe aussi au maintien de la discipline. Les séances de l'après-midi sont calquées sur celles du matin, avec des élèves d'un niveau différent. Par exemple, les plus jeunes récitent leur leçon et en recopient une nouvelle le matin, pendant que les autres revoient la leur; l'après-midi, on permute les groupes : les élèves avancés récitent et 
copient sous la dictée pendant que les plus jeunes continuent la sieste ou apprennent leur leçon du matin.

Les séances bihebdomadaires de récitation générale (subcin) permettent l'apprentissage global du Coran, dans l'ordre croissant de numérotation des sourates. Les enfants se placent autour du maître, en cercle tournant de la droite vers la gauche selon les niveaux décroissants. Le maître annonce le début de la bénédiction en prononçant la parole rituelle : "Rabbahu rabbi laa tadarnii farddan wa anta khayrul waarithiini ». L'élève du plus haut niveau commence la récitation avec le premier verset d'une sourate, les autres élèves finissent de le réciter avec lui (hoorin); un deuxième élève désigné par le maître prend le relais. On finit par la récitation des élèves les moins avancés, la dernière sourate ou l'écriture de l'alphabet. La journée s'achève avec le chant d'une bénédiction (shaafiin).

\section{Approfondissement de l'enseignement religieux}

L'apprentissage coranique nécessite plusieurs années et s'achève quand l'élève est considéré comme un jeune adulte (quinze-seize ans). Ayant obtenu le titre de gardien du Coran, il peut alors ouvrir sa propre école coranique ; il peut aussi choisir de fréquenter une école supérieure religieuse (jamciya). Dèjà avant la colonisation, certains lieux réputés pour la sagesse de leur cheikh dispensaient un enseignement religieux de qualité. À l'époque coloniale, il existait une dizaine de jamciya en territoire somali. Certaines jamciya, placées sous l'autorité d'un ou de plusieurs cheikhs, se trouvaient en ville, au voisinage ou à l'intérieur d'une mosquée. Les autres dépendaient d'une confrérie (dariiqo); toutes possédaient une bibliothèque. Le choix de l'école supérieure était déterminé par la filière souhaitée : la grammaire (naxwa), la langue (lugha) et la littérature arabes étaient enseignées à Tayin, Qulunquul, Harar ou Warsheekh ; la jurisprudence shafi'ite (fiqhi as shafi'i) était la spécialité des jamciya de Mogadiscio, Zeila et Berbera; l'exégèse (tafsir, mais laqmo en Somali) enseignée en langue somali et les Hadith (Paroles du Prophète) celles de Qulunquul, Tayin et Bardheere ; la théologie ou unicité de Dieu (tawhid) celles de Brava et Marka. Dans chacune d'elles, on apprenait aussi le tajwid (manière de réciter le Coran), la conduite des prières et les règles de vie du bon musulman. Les centres religieux se sont multipliés au cours du $\mathrm{Xx}^{\mathrm{e}}$ siècle, en même temps que les communautés confraternelles. Les mosquées se sont aussi largement ouvertes à l'enseignement. Au cours de cette période, les premiers mouvements fondamentalistes et réformistes sont apparus en Somalie.

Ces centres jamciya, en général éloignés du «domicile » des parents, obligeaient l'étudiant (xer) à partir pour de longues années. Ces études religieuses supérieures étaient gratuites, c'est-à-dire sans frais de scolarité ou d'inscription, contrairement aux écoles coraniques élémentaires. Cependant, conduites hors du contexte familial, elles impliquaient des frais annexes 
(hébergement et entretien). Celui qui se rendait auprès d'un cheikh dans une mosquée pouvait subvenir à ses besoins en rendant des services (à un commerçant ou un notable) contre le logement et/ou la nourriture. Le cheikh pouvait aussi solliciter le bon vouloir des personnes fréquentant la mosquée pour garantir au jeune étudiant sans contre-partie une «bourse de nourriture » (jil) : un logement et un à deux repas par jour. Mais il arrivait aussi que celui-ci soit obligé de dormir à l'intérieur même de la mosquée. La situation de l'étudiant religieux était donc souvent difficile, tant moralement que financièrement.

Pour l'étudiant rejoignant une confrérie, elle était assez différente. Les confréries possédaient des terres à l'usage de la communauté, ainsi que des troupeaux, et l'étudiant participait aux travaux collectifs pour dédommagement. Intégré en tant que simple adhérent à la communauté religieuse, qui compte aussi des femmes (car un des devoirs du musulman est de fonder une famille et de procréer), il était soumis alors aux règles édictées par la hiérarchie religieuse de la confrérie. Au fur et à mesure qu'il progressait dans sa formation, il accédait aux fonctions religieuses correspondantes jusqu'à atteindre le niveau le plus élevé (Mohamed-Abdi 1998 : 495). Les étudiants les plus tenaces, formés par un cheikh ou dans une confrérie, se rendaient ensuite dans les pays de la péninsule arabe pour se perfectionner.

Les jeunes gens qui avaient reçu un enseignement religieux jouissaient d'une très haute considération à leur retour chez eux. Aujourd'hui encore, un statut particulier leur est acquis. D'une part, leur initiation, souvent rude car elle les oblige à s'intégrer dans une société qui leur est étrangère, a forgé leur caractère. D'autre part, ils ont acquis un titre religieux leur permettant d'accéder à des fonctions sociales très élevées (conseiller de roi, imam, cheikh, ou encore kadi dans les administrations coloniales), leur assurant en même temps une position économique aisée sinon confortable.

\section{Écoles coraniques traditionnelles dugsi et écoles modernes}

\section{Écoles coraniques et colonisation}

Il est impossible de définir le nombre des dugsi pendant la période précoloniale et coloniale. En effet, parce que l'islam est une religion qui a de tout temps favorisé l'éducation religieuse, et parce qu'il est du devoir de chaque érudit de partager ses connaissances, ces écoles coraniques traditionnelles étaient diversement réparties et fréquentées. Les enfants ou les jeunes gens les quittaient souvent après n'avoir appris que quelques rudiments de langue arabe et les dix premières sourates habituellement utilisées pour la prière (Lewis 1961: 214). Le facteur économique était et reste souvent une contrainte dissuasive. Les filles ne fréquentaient pas du tout l'école, mais tous les garçons n'y étaient pas non plus envoyés. Entre les contraintes économiques (participation des garçons à l'économie domestique), les 
contraintes sociales d'éducation informelle (rites d'initiation des guerriers), et le statut déprécié de ceux qui n'ont pas fréquenté le dugsi ou qui n'y ont pas acquis un minimum de connaissance religieuse, les parents étaient contraints d'opter pour une solution intermédiaire. Le choix pouvait se porter sur un seul des enfants, avec l'espoir qu'il pourrait faire bénéficier ses frères de ses acquis. L'apprentissage était adapté aux conditions de vie : soit condensé pendant les périodes des pluies, quand les familles se déplacent peu (école saisonnière), soit étalé sur de nombreuses années, mais avec une fréquentation partielle de l'école.

Seule une élite parachevait ensuite sa formation dans une jamciya en Somalie puis en Arabie. Des hommes religieux sont ainsi devenus célèbres, dès la fin $\mathrm{du} \mathrm{XIX}^{\mathrm{e}}$ siècle, comme fondateurs de communautés religieuses ou partisans de la lutte anti-coloniale (révolte des Biyomaal et des Derviches), tous ayant pratiqué l'exégèse des textes saints en langue somali ${ }^{3}$.

À partir de la deuxième moitié du XIX $\mathrm{X}^{\mathrm{e}}$ siècle, une mutation s'opère en territoire somali et dans l'ensemble de l'Afrique de l'Est. Cette mutation est l'expression d'une affirmation identitaire qui combine le renforcement des traditions avec celui de l'islam. La revendication de l'appartenance au monde musulman, en opposition aux colons, se manifeste dans l'apparition de noms musulmans en plus grand nombre (Mohamed-Abdi 1990: 9591020). Au cours de cette période, les confréries sont en essor; de nombreuses communautés sont constituées sur l'ensemble du territoire mais plus spécifiquement dans la région agricole fluviale méridionale. Cela entraîne un accroissement du nombre des centres d'études religieuses supérieures. Les écoles coraniques se multiplient elles aussi. À la veille de l'indépendance, plus d'un millier d'entre elles étaient recensées uniquement dans les villes, donnant une éducation religieuse à près de 50000 enfants (Hamdi Al-Sayid Salim 1965 : 489 et sq.).

\section{Les madrasa}

Le terme madrasa désigne une école dispensant en langue arabe un enseignement de religion (Coran), de littérature arabe, de mathématiques (arithmétique) ainsi que d'histoire et de géographie (ces deux dernières n'étant pas enseignées systématiquement). Les élèves sont pourvus de tables et de bancs, ont des cahiers, et l'enseignant utilise un tableau. Comme les dugsi, elle délivre un savoir élémentaire et est ouverte aux enfants et aux adultes, à des horaires différents.

3. Le Coran a été publié en langue somali sous forme partielle à partir des années 1980. Une édition complète du Livre a été achevée en 1988 et publiée en 1991. Le Coran et son exégèse est par ailleurs disponible en somali sur bandes enregistrées (cassettes audio). 
Les madrasa participent de l'affirmation identitaire. Leur nombre s'accroît rapidement, sous l'impulsion des mouvements et des partis indépendantistes (le Somali Youth League notamment). L'attitude des autorités coloniales vis-à-vis des écoles coraniques et des madrasa est assez équivoque. Ces écoles sont tenues en piètre estime quant à leur niveau (Trimingham 1965 : 142). Elles sont aussi suspectées d'entretenir un sentiment xénophobe envers les colons blancs et chrétiens. Pour décourager l'ouverture des madrasa dans le Somaliland, les Britanniques imposent une taxe à leur construction (Mohamed A. Abdulkarim et al. 1978: 65). Dans certaines régions, elles sont interdites et brutalement fermées, et les maîtres sont emprisonnés. Mais dans le même temps (depuis 1929), des subventions sont accordées à des écoles coraniques à condition qu'elles se transforment en madrasa (Lewis 1965 : 100-105). Les autorités coloniales confient certaines fonctions administratives et juridiques de proximité à des érudits musulmans (akils, en somali : caaqil ; kadis, en somali : qaadi), formés dans ces mêmes écoles. Des érudits musulmans sont aussi employés comme enseignants dans les écoles publiques coloniales.

\section{Les écoles coloniales}

Dès 1898, des écoles missionnaires chrétiennes sont ouvertes. Dans le Somaliland, les deux premières sont implantées dans les villes de Berbera et Daymoole, mais leur présence irrite la population et elles sont fermées en 1910 (Lewis 1965 : 103), en vertu d'une loi interdisant le prosélytisme missionnaire ou après l'incident de Daymoole, en 1912 (Mohamed A. Abdulkarim et al. 1978: 58-60). D'autres écoles élémentaires sont ouvertes à partir de 1905, sans grand succès.

Dans la Somalia, ces écoles missionnaires sont mieux perçues : la première est ouverte à Brava (1904). D'autres écoles ainsi que des hospices pour enfants abandonnés et orphelins sont construits (Corni 1937 : 153-164 ; De Vecchi 1935 : 346-352). Les écoles italiennes, fréquentées initialement par les seuls indigènes, accueillent à partir de 1925-1926 des Italiens. En 1929, le nouveau gouverneur décide de séparer les élèves italiens et somali : les premiers recevront alors, dans des classes séparées, une instruction plus magistrale et sanctionnée par un diplôme. Les effectifs autochtones évoluent de façon croissante mais atteignent à peine 2500 en 1928-1929 (De Vecchi 1935 : 350). Entre 1934 et 1938, quelques centaines d'étudiants se sont rendus en Italie pour y recevoir une formation complémentaire (Lewis 1961: 283 ; $\mathrm{MAE}^{4}$ 1961: 95-104).

4. MAE : Ministero degli Affari Esteri ; cela renvoie à l'ouvrage L'amministrazione Fiduciaria della Somalia. 
En $1941^{5}$, la British Military Administration recense alors dans la partie méridionale treize écoles élémentaires pour Somali et Italiens. En 1947, elles sont au nombre de vingt-deux, dont trois ouvertes aux Somali et Arabes pour y recevoir une formation pédagogique (donc d'un niveau supérieur). Dans le même temps, dans le Somaliland (ancien Protectorat), sept écoles élémentaires sont ouvertes et dix-neuf écoles coraniques de type madrasa sont officiellement reconnues et subventionnées. Le parti Somali Youth League (SYL), avec l'approbation de l'administration, ouvre des écoles et des classes en anglais et introduit l'apprentissage de la transcription Osmanya du somali (Lewis 1965 : 123-133) qui fait concurrence à l'arabe.

En fait, dans les deux colonies, les autorités déploient finalement assez peu de moyens pour former la population somali, tant en quantité qu'en qualité, à l'exception des adultes devant fournir du personnel aux administrations (Nelson 1982: 116). D'autre part, jusqu'à la fin de la Seconde Guerre mondiale, l'éducation séculière à l'occidentale n'est pas bien perçue par les Somali.

\section{La préparation de l'indépendance (1950-1960)}

En 1949, l'Italie est mandatée par l'oNU pour administrer le territoire somali et le préparer à l'indépendance. Les régions de l'Ogaden et du Hawd passent sous le contrôle de l'Abyssinie (1948 et 1954). Dans ce contexte de division territoriale réactualisée, l'éducation dispensée aux Somali va suivre des voies divergentes.

Dans les régions sous contrôle abyssin, les relations entre la population somali et les autorités éthiopiennes sont tendues. Les revendications du nouvel État somalien sur les territoires somali (Ogaden et Hawd) et l'attitude irrédentiste de la population empêchent toute politique de développement. L'éducation souffre de cet état de fait : peu d'écoles nouvelles sont ouvertes ; leur fréquentation est rendue difficile par les combats entre les fronts de libération ${ }^{6}$ et l'armée éthiopienne et par l'affrontement direct entre l'Éthiopie et la Somalie (Guerre de l'Ogaden, 1977-1978). Les seules écoles qui parviennent à se maintenir sont les écoles coraniques, du fait de leur mobilité.

5. En 1936, les Italiens, partant de la Somalie méridionale, envahissent l'Abyssinie voisine ainsi que le British Somaliland, chassant les Anglais de leur colonie (1940). Mais en 1941, ceux-ci reconquièrent non seulement leur colonie mais envahissent à leur tour la Somalie italienne et libèrent l'Abyssinie. Tout le territoire somali, à l'exception de Djibouti, est placé sous l'autorité militaire britannique jusqu'en 1950 dans le sud mais jusqu'en 1948 en Ogaden et jusqu'en 1954 dans le Hawd. L'Italie récupère alors son ancienne colonie, sous la forme d'un mandat de l'ONU : c'est l'Administration fiduciaire italienne de la Somalie (AFIS).

6. Ogaden Liberation Front, 1963-1970 ; Western Somalia Liberation Front, 19701990 ; Ogaden National Liberation Front, constitué à la fin des années 1980 et encore actif aujourd'hui. 
Depuis 1990, la reconnaissance des nationalités a donné un nouveau souffle à l'éducation : les matières sont enseignées en langue somali par des enseignants somali.

L'administration fiduciaire de la Somalia Italiana s'applique à promouvoir l'éducation et à préparer le pays à l'indépendance. Avec l'aide de l'Unesco, des programmes scolaires pour les niveaux primaires, élémentaires (collèges) et secondaires (lycées), pour enfants et adultes, sont définis. Des lycées professionnels (pêche, industrie, agriculture) et techniques (commerce, administration, économie) sont ouverts. Dans le Somaliland, en revanche, et malgré la création d'un comité pour l'Éducation (1954), seuls deux lycées pour garçons sont ouverts (1953 et 1959), une école pour fille (Burco 1953) et une école de commerce (1952) (Lewis 1961 : 148-149).

Au cours de cette période, les Somali, par l'intermédiaire de leurs partis politiques indépendantistes, demandent l'aide du gouvernement égyptien, notamment dans le domaine de l'éducation. Après la prise de pouvoir par Nasser, des écoles publiques égyptiennes sont ouvertes dans les principales villes de la Somalia. Ces écoles sont gérées par le gouvernement égyptien qui fournit matériel scolaire (manuels) et enseignants. Les programmes et les matières, enseignées en langue arabe, sont identiques à ceux des écoles d'Égypte. Les études sont sanctionnées par les mêmes diplômes (Mohamed A. Abdulkarim et al. 1978 : 64-80 ; Lewis 1961 : 289). L'engouement pour la langue arabe n'est pas nouveau et s'inscrit dans la logique du processus identitaire. Une demande solennelle (1950) d'officialisation de la langue arabe est faite par l'ensemble des chefs (séculiers et religieux) et des représentants des partis somali auprès des autorités italiennes. Elle est dès lors enseignée dans les écoles publiques. Les journaux indépendantistes paraissent en langue arabe, à défaut de pouvoir l'être en langue somali.

À la veille de l'indépendance, la situation scolaire montre des disparités importantes. Les écoles publiques coloniales et les écoles privées de langue arabe, aussi bien dans le Nord (Somaliland) que dans le Sud (Somalia Italiana) n'ont pas de programme commun et ne préparent pas à des diplômes équivalents.

Au niveau élémentaire, le déséquilibre est tel que les écoles coraniques fixes sont cinq fois plus nombreuses que les écoles élémentaires publiques, ouvertes aux Somali et aux enfants de colons ; ce déséquilibre est beaucoup plus important dans le Sud que dans le Nord (Hamdi Al-Sayid Salim 1965 : 489 et sq. ; Mohamed A. Abdulkarim et al. 1978 : 72). Ces différences d'implantation des écoles coraniques et des écoles élémentaires publiques s'expliquent par les perceptions de l'éducation : les écoles coraniques s'intègrent dans la culture somali alors que les écoles coloniales sont des « pièces rapportées » peu appréciées, même si elles permettent d'accéder à des formations complémentaires et des emplois (fonctionnariat, ouvriers spécialisés). Les madrasa tiennent une place à part car elles combinent enseignement religieux «intégrateur » et enseignement laïc. La situation scolaire présente aussi des écarts importants dans les deux colonies. Les écoles sont plus 
nombreuses dans la Somalia que dans le Somaliland. Les effectifs indiquent des tendances encore plus marquées: les écoles coraniques méridionales totalisent un effectif 14 fois supérieur à celui des mêmes écoles dans le Nord. Par conséquent, les écoles sont globalement plus chargées dans le Sud, qu'elles soient publiques (coloniales) ou privées (coraniques). Plusieurs observations permettent d'expliquer ce clivage. La Somalia, et la région fluviale en particulier, est deux à trois fois plus peuplée que le Somaliland, avec une proportion de sédentaires plus élevée. Le Nord, plutôt aride, est tourné vers l'élevage extensif «nomade » alors que le sud combine élevage «nomade » et agriculture fixe. De ce fait, les confréries religieuses se sont plutôt implantées dans le Sud, d'où la multiplication des écoles coraniques sédentaires. Par ailleurs, les deux colonies ont développé des politiques éducatives différentes. Dans le Somaliland, les écoles, de tous niveaux, sont en majorité des internats et sont donc de coût élevé. Seule une élite, par ailleurs convaincue de l'intérêt de l'éducation à l'occidentale, possède les capacités matérielles d'en assumer les frais. Dans le Sud, en revanche, les écoles n'ont pas d'internats, sauf quelques exceptions. De plus, les autorités italiennes ont mené une campagne de sensibilisation à l'éducation et ont bénéficié, pendant la période de tutelle, du soutien de l'Unesco.

\section{Période civile (1960-1969) et militaire, développement et régression de l'éducation}

Lors de l'indépendance (26 juin et $1^{\text {er }}$ juillet 1960), le gouvernement de la nouvelle République de Somalie unifiée est confronté à une situation scolaire complexe. Une querelle sur le choix d'une langue officielle (administration, justice, enseignement) oppose partisans de la langue arabe et de la langue somali.

La politique d'éducation du gouvernement civil n'est pas clairement définie. Entre 1960 et 1969, les écoles héritées de la colonisation ont diminué en nombre ; celles établies dans les campagnes, coûteuses car peu fréquentées, sont fermées, mais entre temps, les effectifs ont doublé. Le schéma se répète au niveau des écoles intermédiaires (collèges), secondaires (lycées) et de la formation pour adultes. Seules les écoles privées, coraniques, madrasa, égyptiennes ou missionnaires, continuent de se développer : $31 \%$ des effectifs sont inscrits dans le privé en 1969.

Les militaires, au pouvoir depuis octobre 1969, convaincus du rôle de l'éducation moderne dans le développement, adoptent une politique plus volontaire (Voelter 1984 : 288). Le somali est officialisé et un comité est désigné pour en choisir une transcription. En 1973, une première promotion est formée à la transcription en caractères latins (disponible depuis 1972). En 1974-1975, les écoles secondaires sont fermées pour cause de campagne d'alphabétisation. Tous les jeunes et les enseignants sont envoyés dans les villes, les villages, les campements nomades pour enseigner l'écriture et la 
lecture du somali. À la fin de la campagne, et sans avoir reçu aucune aide extérieure, la Somalie compte 1757779 personnes alphabétisées (MION ${ }^{7}$ 1980 : 38). Des manuels sont édités en langue somali et des efforts importants sont faits pour élargir le somali à la terminologie scientifique.

Les écoles privées sont nationalisées, les programmes scolaires uniformisés et l'enseignement dispensé en somali est obligatoire et gratuit dans le primaire. Des examens nationaux sanctionnent la fin de chaque cycle (préprimaire, primaire, secondaire et supérieur). Le premier niveau correspond à l'école coranique. Certains n'intègrent l'école qu'après avoir achevé l'apprentissage du Coran. Un examen d'entrée permet d'évaluer leur niveau et donc la classe d'intégration. Une autre possibilité, puisque l'école d'État n'a lieu que le matin ou l'après-midi, est de continuer l'apprentissage du Coran en alternance (cours de l'après-midi ou du matin). Le primaire est le cycle le plus long ( 8 ans) et regroupe les niveaux élémentaire et intermédiaire (du système anglais), le passage du premier au second niveau ayant été rendu automatique.

L'examen de fin de primaire sanctionne l'entrée au lycée d'enseignement général (4 ans), technique ou professionnel (3 à 4 ans), également gratuits. Les langues étrangères (anglais et arabe) sont enseignées à partir de la cinquième année du primaire. En revanche, l'italien n'est appris qu'après le lycée, en « cours intensif » de six mois avant l'entrée en faculté. L'université est majoritairement financée par le gouvernement italien qui fournit aussi les enseignants (Voelter 1984 : 294-295). En 1974, la Somalie devient membre de la Ligue arabe et l'arabe devient la deuxième langue officielle du pays. Son apprentissage est rendu obligatoire (1977) du primaire à la fin du secondaire (Lujna 1986 : 32) et de nouveaux programmes sont établis en 1979 visant à renforcer l'usage de la langue arabe (épreuve obligatoire pour la poursuite des études).

Mais après des débuts prometteurs, le système éducatif se dérègle à partir de la Guerre de l'Ogaden (1977-1978) qui a vidé les caisses de l'État (Nelson 1982 : 281 ; Nations Unies 1997). Par ailleurs, la montée des mouvements armés d'opposition, à partir des années 1980, a un effet néfaste sur l'économie du pays. La politique intérieure change et le système éducatif connaît une crise laminaire : les salaires des enseignants ne leur permettent pas d'assurer le minimum vital pour eux-mêmes et leur famille, et les dépenses de l'État pour le renouvellement des manuels, des livrets pour les enseignants et du matériel technique sont dérisoires (Retamal \& Devadoss 1998 : 74-75). Par ailleurs, un nombre toujours croissant de personnes sans qualification sont nommées, par le jeu des alliances claniques, à des postes parfois élevés et bien rémunérés. Les enseignants, obligés de recourir à d'autres emplois, désertent leur poste ou ne l'occupent que de façon partielle. Locaux et mobiliers scolaires se délabrent. La fréquentation de l'École devient épisodique. La dégradation est telle qu'en 1990, et en comparaison

7. MION désigne le ministère de l'Information et de l'Orientation nationale somalien. 
avec 1982, une école primaire sur quatre avait fermé ses portes. De fait, les jeunes sont marginalisés par la société : les anciens savoirs ne sont plus transmis parce qu'ils sont démodés, les nouveaux ne servent plus à rien par manque de débouchés, sauf à faire jouer des relations personnelles. La petite délinquance, liée à la consommation d'alcools forts, de drogue (khat) et à la prostitution, devient pratique courante. Les rues ne sont plus sûres. La prise en charge de l'éducation des jeunes n'est pas assumée par les parents, eux-mêmes préoccupés par la recherche de moyens de subsistance, ni par la communauté des adultes destituée de ce rôle dans les villes. C'est le processus de mooryanisation ${ }^{8}$ des jeunes qui deviennent, au cours de la guerre civile, les machines à tuer et la « chair à canon» des chefs de guerre. Seules les écoles coraniques, qui se sont ouvertes aux filles, perdurent pendant cette période et se développent sans percevoir aucune subvention de l'État alors que les écoles primaires, intermédiaires et secondaires se vident de leurs élèves. Elles sont les seules capables d'assumer un rôle éducatif et intégrateur vis-à-vis des jeunes, rôle grandement renforcé par l'attitude des parents. Cependant, beaucoup d'enfants ne les fréquentent plus, passé un certain âge.

\section{La scolarité aujourd'hui}

La guerre civile a éclaté en 1990, a détruit toutes les infrastructures (locaux scolaires endommagés ou détruits, matériel scolaire pillé) et a provoqué le déplacement des enseignants. Pendant près de trois ans, jusqu'en 1993, la plupart des écoles publiques des grandes villes ont cessé de fonctionner. Les combats et les déplacements massifs de population ont provoqué de nombreux bouleversements. Les familles ont dû se recomposer en essayant de faire face aux traumatismes directs et indirects. Cependant, des structures éducatives de type moderne sont réapparues. Leur prise en charge a été réalisée par la population, par des ONG ou des agences internationales, et la présence de l'onusom a facilité cette renaissance. Ainsi, à Mogadiscio, 24 des 85 écoles que comptait la ville en 1990 ont été réhabilitées en 1995 (UNESCO-PEER 1995: § 7). Dans certaines régions, des comités se sont constitués pour réouvrir des écoles et établir des programmes. D'anciens locaux ont été réparés et transformés en salles de classe et de nouveaux bâtiments ont été construits pour accueillir les élèves, avec la participation active de la population et d'enseignants. D'anciens manuels scolaires et des guides pour les enseignants, existant déjà avant 1990, ont été réédités par les

8. Le terme mooryanisation est un néologisme construit à partir du somali mooryan, utilisé pendant la guerre civile pour désigner les jeunes gens (très souvent de moins de vingt-cinq ans) devenus «soldats » ou miliciens des chefs de factions. Mooryan signifie «démuni de morale et en même temps de biens matériels »; c'est aussi le nom d'une espèce de fourmi noire qui se déplace en colonne de milliers d'individus en détruisant tout sur son passage. 
agences des Nations Unies (UNICEF et al. 1999: 15) qui ont aussi implanté des écoles primaires dans les camps de réfugiés (Djibouti, Kenya, Éthiopie) en garantissant aux enseignants des salaires en nature (nourriture) et, pendant une période, en distribuant des rations alimentaires aux enfants. Dans quelques villes, des cycles complets ont pu être rétablis. Dans le Nord, une administration supervisant l'Éducation a été mise en place et l'enseignement, harmonisé, a repris l'ancien modèle du trois fois quatre ans (primaire, intermédiaire, secondaire).

Mais cette situation est loin d'être générale. Un rapport (UNICEF et al. 1999) évoque la diversité des enseignements dispensés dans le primaire, liée aux différentes ONG et agences œuvrant en Somalie, et même si des programmes ont pu être coordonnés, ils divergent d'une région à l'autre, d'une ville à l'autre, tant par la langue d'enseignement (somali, anglais, arabe) que par les manuels disponibles. La cohésion est absente des cycles puisque certains sont calqués sur le modèle somalien des trois fois quatre ans, d'autres sur le modèle arabe ( 6 ans pour le primaire, 3 pour l'intermédiaire et deux pour le secondaire).

En 1997, 773 écoles primaires ont été recensées dans l'ensemble du pays, dont 131 dans la seule région du Benadir (63 à Mogadiscio) (UNDOS 1998 : 51-53). Seulement 613 d'entre elles fonctionnent réellement (UNDP 1998 : 71-73). L'effectif global stagne autour de 150000 élèves. Dans les premières classes (niveaux 1 à 4), les effectifs sont importants, mais à partir de la quatrième (première année du cycle intermédiaire), ils chutent brutalement. Globalement, un enfant sur cinq est scolarisé dans le primaire ou l'intermédiaire, et bien souvent, il est en retard. Il semblerait par ailleurs que Mogadiscio bénéficie d'une situation privilégiée puisque les enseignements secondaire (lycée) et supérieur (université) y sont assurés. Des particuliers, avec des moyens financiers appropriés, ont accès à des écoles privées payantes qui préparent des élèves d'un très bon niveau. Certains enfants peuvent aussi bénéficier de précepteurs.

Les écoles coraniques ont continué de fonctionner tant bien que mal, en dépit de la dégradation de la situation. Au cœur même des combats et sous les bombes, des macallin faisaient la classe et des enfants récitaient le Coran, payant parfois de leur vie leur ténacité. Dans les camps de réfugiés à l'extérieur du pays (au Kenya, en Éthiopie) et les camps de déplacés à l'intérieur, ces écoles ont poursuivi leur mission éducative. Au plus dur de la guerre civile, elles ont aussi servi de refuge aux enfants et à leurs mères, et grâce à des dons extérieurs et à des ONG musulmanes, elles ont joué le rôle de centres de nutrition. Les écoles coraniques sont restées des écoles de proximité, par exemple, «dans le Somaliland, il y a une école coranique tous les 300-500 mètres parmi les populations sédentaires » (UNDP 1998 : 77). Les cours y durent généralement moins d'une demi-journée. À la fin des années 1990, elles représentaient dans les régions rurales ou à population nomade, quasiment la seule possibilité d'instruction. Ouvertes aussi aux filles, elles éduquent de 40 à $60 \%$ des enfants de la tranche d'âge 6-14 ans 
(UNICEF et al. 1999 : 22) et dans les zones urbaines, plus d'un tiers des enfants scolarisés fréquentent à la fois une école coranique et une école primaire en alternance. Cependant, une grande majorité des enfants en âge d'être scolarisés ne le sont pas.

De toutes les structures scolaires ayant existé en territoire somali depuis le début de ce siècle, les écoles coraniques sont les seules à avoir maintenu une continuité pendant la guerre civile. On peut arguer que leur persistance est liée à leur infrastructure légère. La pénurie des enseignants coraniques ne s'est pas faite sentir de la même façon que celle des enseignants laïcs. En effet, tout homme ayant achevé l'apprentissage du Coran est apte à l'enseigner à son tour. L'école coranique est socialisante; elle fait partie du paysage culturel. Les enfants, jusqu'à l'adolescence, sont pris en charge par la communauté. Après avoir représenté une forme d'affirmation identitaire pendant la période coloniale, l'École coranique aujourd'hui exprime plutôt un repli sur soi, « un retour aux sources » de la société somali dévastée par la guerre civile.

Il existe un risque que ce repli devienne un engagement dans la voie des fondamentalismes religieux musulmans. Déjà certaines régions sont soumises à la loi islamique appliquée dans son sens le plus strict. Les mouvements intégristes occupent aussi la scène politique et font opposition aux factions armées. On peut donc s'interroger, dans ce contexte, sur la signification de l'aide apportée dans le domaine de l'éducation par certains pays musulmans.

Le maintien de l'École coranique s'explique aussi par les défaillances du système éducatif public et laïc. Après avoir connu une période faste (entre 1969 et 1980 environ), celui-ci s'est progressivement dégradé. À quoi bon envoyer un enfant à l'École d'État si celui-ci n'est pas en mesure de lui assurer un avenir, c'est-à-dire un travail et un salaire décents ? Aujourd'hui, seule une minorité de jeunes ont accès à une forme d'éducation et celle-ci reste très limitée tant dans la durée d'études que dans le niveau de formation atteint.

Dans l'hypothèse d'un retour à la paix et de la reconstruction d'un État, le problème de cette génération "gâchée par la guerre civile », et qui n'aura reçu aucune formation ni aucune instruction, est crucial. Quelle place pourra-t-elle tenir dans la société, quel rôle social et politique pourra-t-elle jouer? La reconstruction de l'État risque de s'avérer impossible sans le personnel qualifié nécessaire. La remise en route d'un système éducatif performant, pouvant répondre aux besoins d'un pays en reconstruction, sera longue car la priorité sera de former les enseignants nécessaires à sa constitution. Pour combler partiellement le vide institutionnel actuel, l'Unicef a envisagé la solution de se servir des écoles coraniques pour élargir le champ 
d'action des écoles primaires et secondaires. Il s'agirait, en quelque sorte, de les transformer en madrasa, c'est-à-dire en écoles combinant enseignements religieux et séculier. Toutefois, la question de la perception de cet enseignement séculier par la société et les parents demeure. Souvent jugé non conforme à la morale et aux traditions, cet enseignement laïc doit être repensé en fonction des mentalités et des besoins. C'est son image positive auprès des parents et de la communauté qu'il faut rétablir et son utilité ne sera reconnue que s'il débouche sur une amélioration des conditions d'existence et du niveau de vie de chacun.

Institut de recherche pour le développement, Bondy.

\section{BIBLIOGR APHIE}

AHMED Ben 'AbD El-QÂDER (surnommé ARAB-FAQIH)

s.d. Histoire de la conquête de l'Abyssinie. Trad. par R. Basset, Paris, Leroux.

CORNI, G.

1937 Somalia Italiana, 2 vol., Milan, Editoriale Arte e Storia.

DE VECCHI, C.

1935 Orizzonti d'Impero, cinque anni in Somalia, Milan, A. Mondadori.

Hamdi Al-Sayid SALim

1965 Al-Somal, qadiman wa hadithan (La Somalie ancienne et contemporaine), tome 2, Mogadiscio, Ministère somalien de l'Information.

LEWIS, I. M.

1961 A Pastoral Democracy, London, Oxford University Press.

1965 The Modern History of Somaliland, from Nation to State, New York, Frederick Praeger Publishers.

LujNA (Lujna al wataniya al uliya li hamlat taqwiyati al luqa al arabiya)

1986 Al nadwa al cilmiya hawla hamlat taqwiyat al luqa al arabiya (colloque scientifique autour de la campagne de renforcement de la langue arabe), Muqdisho, LUJNA.

MAE (Ministero degli Affari Esteri)

1961 L'amministrazione fiduciaria della Somalia, Rome, Istituto poligrafico dello Stato. 
MeUnier, O.

1995 «Enseignements de base, politiques d'éducation et stratégies éducatives en milieu haoussa. Le cas de la ville de Maradi (Niger)», in M.-F. LANGE \& J.-Y. MARTIN (dir.), Les stratégies éducatives en Afrique subsaharienne, La Tour D'Aigues, Éditions de l'Aube-IRD (Autrepart, Cahiers des sciences humaines, 31 (3)) : 617-634.

1997 Dynamique de l'enseignement islamique au Niger, Paris, L'Harmattan.

MION (Ministère de l'Information et de l'Orientation nationale)

1980 Al-taqadum al-iqtisadi fi al-Somal (Les progrès économiques en Somalie), Mogadiscio, Ministère de l'Information et de l'Orientation nationale.

Mohamed A. Abdulkarim, Abdulkadir Sh. Abdulahi, Abdulqakir Sh. Yusuf, Omar A. Ahmed \& Ahmed J. Mohamed

1978 Tariikh al-ta'lin fi al-Somal (Histoire de l'enseignement en Somalie), Mogadiscio, Ministère de l'Éducation.

Mohamed-ABdi, M.

1990 La Somalie aux hautes périodes, Thèse de Doctorat, Besançon, Université de Franche-Comté.

1998 «De l'Islam traditionnel à l'Islam intégriste», in M. MoHAMED-ABDI, Recherches sur une société riche d'une structure complexe : la société somalie (mémoire de HDR), Besançon, Université de Franche-Comté : 481-543.

NATIONS UNIES

1997 Statistical Yearbook, New York, Nations Unies.

Nelson, H. D.

1982 Somalia, a Country Study, Washington D. C., Department of the Army.

Retamal, G. \& Devadoss, M.

1998 «Education in a Nation with Chronic Crisis : the Case of Somalia », in G. Retamal \& M. Aedo-Richmond (eds), Education as a Humanitarian Response, Unesco-Peer : 74-93 (polycopié).

Tourneux, H. \& IYÉBi-MANDJEK, O.

1994 L'école dans une petite ville africaine (Maroua, Cameroun), Paris, Karthala.

TRIMINGHAM, J. S.

1965 Islam in Ethiopia, London, Frank Cass \& Co.

UNDOS

1998 Regional Profile. Mogadishu/Benadir, Nairobi, UNDOS.

UNDP (United Nations Development Programme)

1998 Human Development Report, Somalia 1998, s. 1., UNDP.

UNESCO-PEER

1995 Rehabilitating Somalia's Education Sector, Paris, polycopié.

19991998 Annual report, Nairobi, UNESCO-PEER. 
UNICEF Somalia, UNDP/UNFPA-Somalia, UNESCO Somalia

1999 Somalia: Year 2000 Education for all Assessment Report.

VOELTER, W.

1984 «Primary, Secondary and Third Level in Education in Somalia», in T. Labahn (ed.), Studies in Humanities and Natural Sciences, Proceedings of the Second International Congress of Somali Studies, vol. 4, Hamburg, Helmut Buske Verlag : 287-297.

\section{RÉSUMÉ}

Cet article traite de l'évolution de l'enseignement pratiqué en Somalie au cours du $\mathrm{xx}^{\mathrm{e}}$ siècle (périodes précoloniale, coloniale, gouvernements civil et militaire, guerre civile). II décrit en particulier le fonctionnement des écoles coraniques et leur importance dans le système éducatif somali traditionnel. Malgré le développement des écoles laïques, les dugsi se sont maintenues ou ont évolué vers des écoles madrasa (enseignement laïc et religieux). Après l'éclatement de la guerre civile, elles ont continué de fonctionner et d'assurer leur rôle éducatif auprès des enfants.

\section{ABSTRACT}

Back to Koranic Schools in Somalia. - The education dispensed in Somalia during the 20th century has varied from the precolonial to the colonial period in the context of civilian and military governments, and the civil war. The operation of Koranic schools is described along with their importance in the traditional system of education. Despite the development of secular schools, the dugsi still exist since the civil war and have changed toward becoming seminaries providing religious and nonreligious instruction.

Mots-clés/Keywords: Somalie, colonisation, École coranique, éducation, dugsi, enseignement laïc, guerre civile, madrasa/Somalia, colonization, Koranic schools, education, dugsi, secular education, civil war, madrasa. 